\title{
Interactive Smart Space for Single-Person Households Using Electroencephalogram through Fusion of Digital Twin and Artificial Intelligence
}

Seung Yeul Ji (D)

Citation: Ji, S.Y. Interactive Smart Space for Single-Person Households Using Electroencephalogram through Fusion of Digital Twin and Artificial Intelligence. Energies 2021, 14, 7771. https://doi.org/10.3390/en14227771

Academic Editor: Gianpiero Colangelo

Received: 30 September 2021

Accepted: 16 November 2021

Published: 19 November 2021

Publisher's Note: MDPI stays neutral with regard to jurisdictional claims in published maps and institutional affiliations.

Copyright: (C) 2021 by the author. Licensee MDPI, Basel, Switzerland. This article is an open access article distributed under the terms and conditions of the Creative Commons Attribution (CC BY) license (https:/ / creativecommons.org/licenses/by/ $4.0 /)$.
School of Architecture, Hanyang University, Seoul 04763, Korea; musicji83@hanyang.ac.kr; Tel.: +82-2-2220-1796

\begin{abstract}
The core technology for building a smart space includes the capability to analyse the space for users using various sensors. The purpose of this study was to propose a personalised interactive smart space implementation model driven by the fusion of digital twin (DT) and artificial intelligence (AI) based on electroencephalogram (EEG) data. This study utilised a handheld EEG sensor to identify a user's emotion information and focused on the connection with the space. A smart space for single-person households that responds to EEG-based biometric information was designed for an interactive space that can improve the current emotional state of the space user. The technical characteristics of DT and AI were analysed to control spatial changes according to the user's emotional state and to address safety-related issues. Furthermore, a fusion mechanism for DT and $\mathrm{AI}$ was developed for intelligent motor control to change the dimensions of the space in order to improve the EEG state of the user. In addition, using an AI model that converts EEG data into emotional state information, the user's emotional state was analysed, and key issues related to the spatial dimensions and change of space that induce psychological stability were investigated.
\end{abstract}

Keywords: smart space; smart environment; digital twin (DT); electroencephalogram; artificial intelligence

\section{Introduction}

\subsection{Background}

With the advent of the 4th Industrial Revolution, various approaches to introduce smart systems to existing architectural spaces have been proposed [1]. Smart space refers to ensuring user convenience through communication between humans and physical space using digital technology [2]. For such "smart spaces", the core technology includes the analysis [3] of the user space using various types of sensors [4].

Traditionally, user behaviour analyses have been conducted using observational methods and surveys [5]. However, with such information, arbitrary filtering occurs when the user jots down their thoughts explicitly, which may lead to inaccuracies [6].

Therefore, to supplement such analyses and obtain objective data, researchers have attempted to develop a fundamental approach for determining user emotions through electroencephalogram (EEG) sensor data, which is the stage preceding perceptual interpretation [7].

Eberhard argued that research on EEG [8] will lead to an improved understanding of how the design method in architecture can be changed. Eberhard assumed that a better understanding of the impact of buildings and space on the mental state of the users will also give rise to a better understanding of the mechanism of activities in the human brain. He also described the architectural design standards of schools, offices, laboratories, memorials, churches, and facilities for the elderly, and presented hypotheses on human experiences in these environments.

Gero [9] hypothesised that an open space is more effective than a constrained space for creativity and compared EEG analysis results with the assessments of 18 professional industrial designers to identify the correlation between the sense of openness in space and 
brain activation, using a protocol analysis method. Although a number of experts and researchers have conducted research to monitor a user's EEG in an architectural space, analyse the correlation between EEG results and the space, and to verify the hypothesis, there have been no reports on the interactions required to achieve psychological stability through changes in the architectural space using EEG data.

Therefore, this study aimed to collect and collate EEG information of a user in an architectural space environment and utilised this information as data indices to investigate the optimal psychological space for the user. As opposed to existing studies, this study also aimed to induce psychological stability in a user by controlling the space based on EEG information.

\subsection{Methodology}

The information on the emotion that the user experiences in an architectural space was collected through EEG. Moreover, for interactive changes in the space for composition of positive space, a digital twin (DT) that connects virtual digital space with physical space using biometric information was utilised as the key technology.

With regard to the configuration of the smart space system, the scope of this study was set to include spatial optimisation via spatial expansion and shrinkage based on stress and engagement among the EEG sensor data of the user (Figure 1).

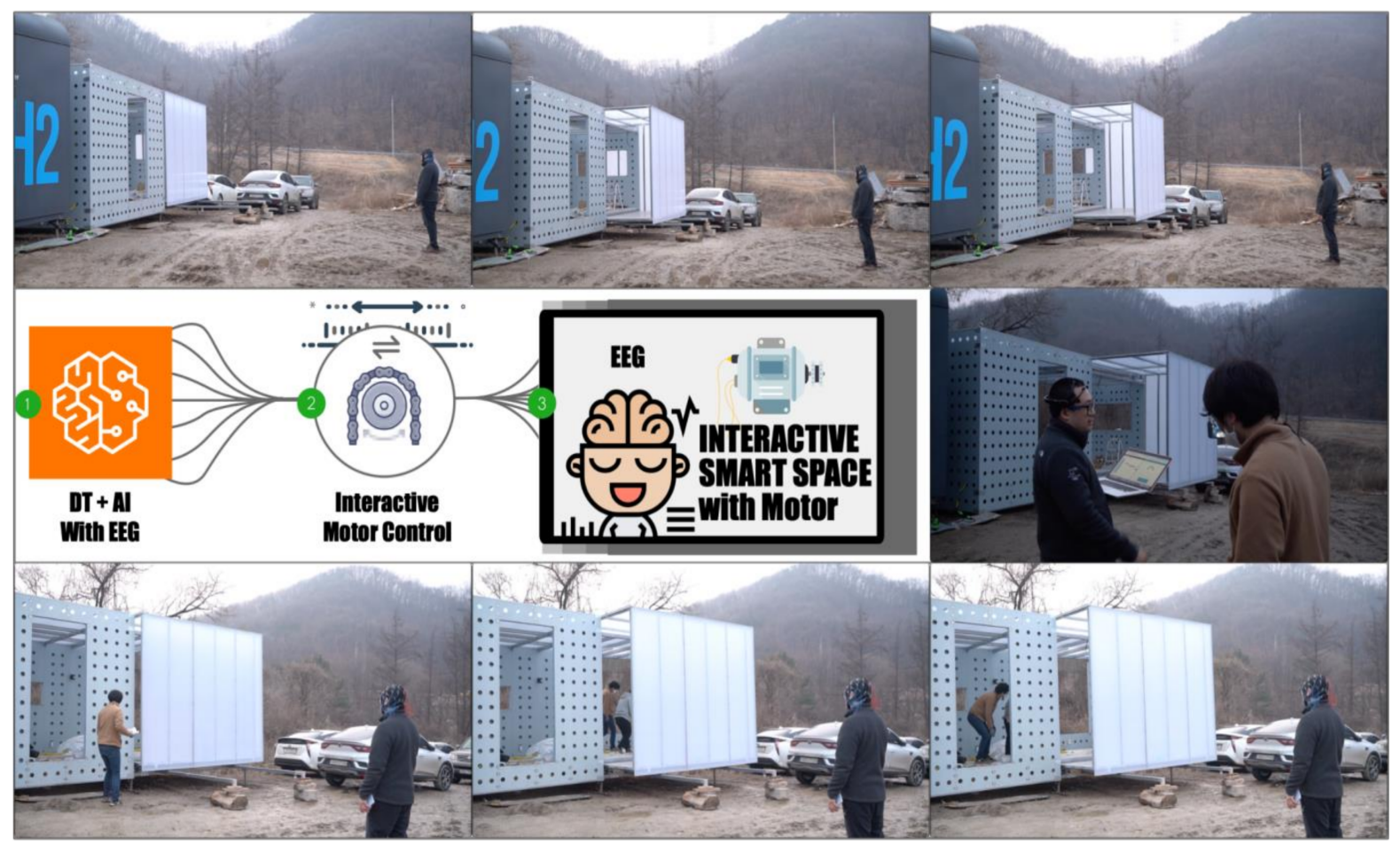

Figure 1. Smart space operation with EEG.

To conduct the experiment (8 January 2021), the KIRD (Korea Institute of Human Resources Development in Science and Technology)-hosted research ethics education program was undertaken; the certification code is 2021-01-31-S-E-007979.

The study was conducted as follows:

- $\quad$ First, we explored the fusion mechanism of DT and AI for a smart space that induces a stable psychological state and acts as the core technology for driving a linear actuator motor for interactive control of the space through theoretical analysis.

- Second, virtual sensors in a game engine and real physical sensors for user safety were used, and an agent model for the changes according to EEG information with the spatial dimension control system was built based on a deep learning algorithm. The 
performance optimisation factors of the motor control system were analysed according to EEG references, and the space control process was controlled using an interactive motor composed of a linear actuator.

- Third, we developed a personalised smart space implementation model that is driven by the fusion mechanism of DT and AI based on the user's EEG data as part of a task in which the machine operation is affected by the human mind.

\section{Theoretical Analysis}

\subsection{Integrated Interactive System of DT and AI for Smart Space}

An interactive motor control centre operated via the fusion of DT and AI was constructed to develop an interactive motor control model of the smart space; the aim was to provide the optimal psychological space for the user and to optimise the performance of the system that reflects the EEG information. The AI control centre of the system, which reflects the EEG information, is shown in Figure 2. The essence of the system that reflects EEG information is the smart space control, which considers the emotional information of the user. In this system, analysis of the stress index loss before and after the change in spatial dimension using the DeepFM algorithm is important, along with the capability to detect abnormalities through EEG data feedback for optimisation of the mechanical motor and its control. Therefore, the integrated interactive system of DT and AI with a continuously improving abnormality detection function plays an instrumental role in smart space control.

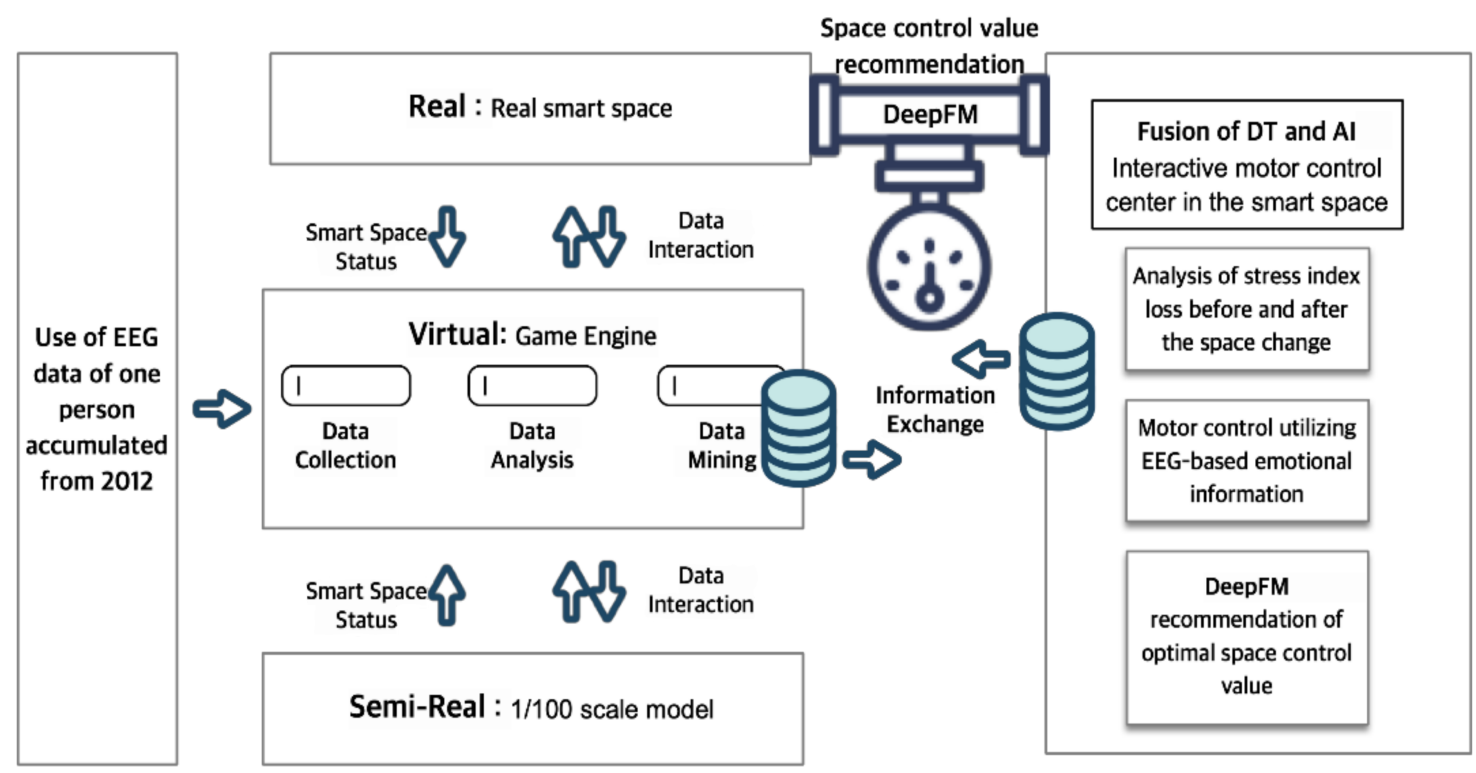

Figure 2. Integrated interactive system of DT and AI for a single user in a smart space.

\subsection{EEG Signal Process in Smart Space and Implication of Delay}

In the experiment, the space size was changed through space transformation for stable induction of the user's stress index and engagement, using an AI-based recommendation system model and by collecting EEG information from users in the smart space.

As shown in Figure 3, the EEG signals were collected from channel-specific raw data of the experimenter wearing the EEG equipment in the smart space, and the alpha, beta, theta, delta, and gamma waveforms were extracted using an FIR (Finite impulse response) filter. Through feature analysis of each waveform, the user's emotion information was derived using the Emotiv AI model; this information was then converted to data corresponding to engagement, stress, relaxation, excitement, and interest. The emotion information was utilised in DeepFM - an AI model to control the size of a smart space-and was applied to the smart space. However, to improve the accuracy and performance of the 
model for application in smart spaces, since 2012, we have been conducting an EEGbased architectural space experiment and transfer learning using EEG profile data, which represents pattern histories for the EEG data collected; accordingly, the record data for training was expanded. In this experiment, the building moved while the user was directly located inside the building; this allowed for adjusting the size of the space using the EEG data. Therefore, in consideration of safety, the first delay occurs, which provides the user's emotional information from the EEG equipment as a safety device through physical simulation of the game engine and procedural verification of the $1 / 100$ scale model. This is followed by the second delay, in which DeepFM is utilised to receive a recommendation for a varying smart space using the extracted emotional information. Based on the pathological standard of Chombard de Lauwe, which pertains to an individual's psychological state and architectural space size, the space to induce a stable EEG state was transformed by deriving the characteristic points of emotional information and by using a relationship that expands the space when the stress is high and reduces the space when the engagement is low (Figure 3).

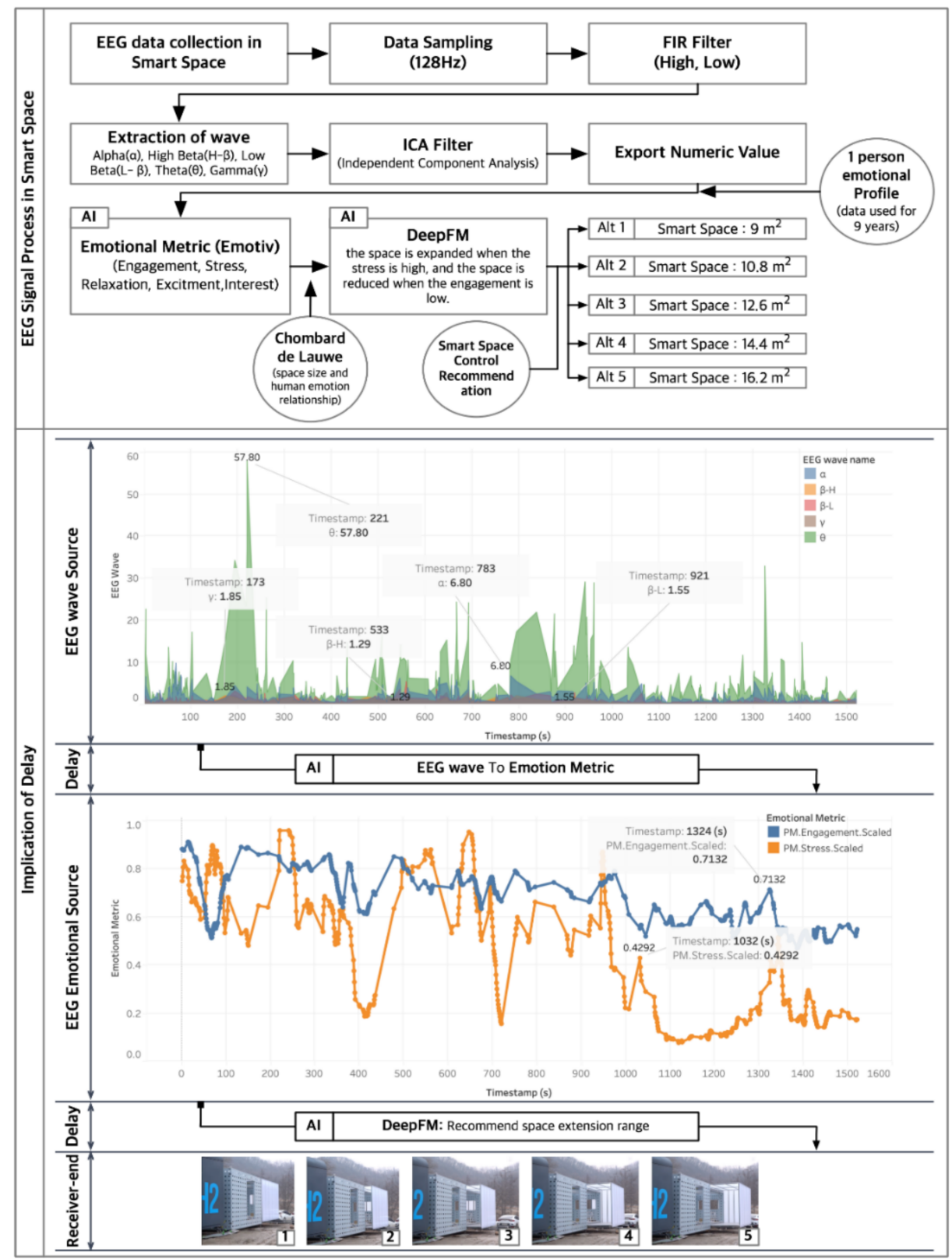

Figure 3. EEG signal process in smart space and implication of delay. 


\section{Integration of DT and AI Based on EEG for Intelligent Motor Control \\ 3.1. Fusion Mechanism of DT and AI}

The essence of smart space construction driven by the fusion of DT and AI is a twin model of virtual space, and the purpose is to build an optimal space considering the emotional state of the space user. To this end, the rules for operating the smart space were set based on the geometric model linkage process, physical model, and sensor data. Through virtual model simulation using a game engine, interactive space elements are visualised and digitised to provide a data feedback process between the user's EEG information in the physical space and the virtual model in the game engine; this ensures that the aforementioned process will be executed simultaneously with the interactive system. Thus, the information of the virtual space is reflected in the real space, the model in the virtual environment is controlled based on the sensor information in real space, and data are collected and collated. In particular, the integration of AI-based deep learning and the user's biometric information enables intelligent coordination of the operation and control of the interactive space system as well as intelligent diagnosis of the databased system and the prediction of psychological stability after operation. Consequently, coordination and control were performed through a physical space change system and through data feedback for six types of EEG channel information; moreover, an interactive smart space model with optimisation effects for deep learning and emotional information was developed in this study.

Figure 4 illustrates the mechanism for the fusion of DT and AI by applying Gartner's DT data [10]. The basic process of this mechanism is that the environmental information of the space for the element information for the operation of the actual space structure is collected, and the dynamic state of the space is detected through the sensor. In this study, using a game engine-based 3D virtual model and a 1/100 scale model, information such as the spatial environment of the real building and mechanical properties for interactive control was collected, and the dynamic state was detected to provide the foundation for the structural setting for DT and AI analysis. The deep learning model-based biometric data information management and control platform serves as the core of DT and AI technology integration. The intelligent space management and control platform formed by the fusion of the two technologies includes the following two main components: judgement for the biometric information of the AI algorithm and the DT model. For the efficient operation of the smart space system, DT and AI consist of two parts: biometric information management and control. Based on emotion information, intelligent diagnosis of the variable space system and prediction method of emotional information before and after space change form the core of the AI process.

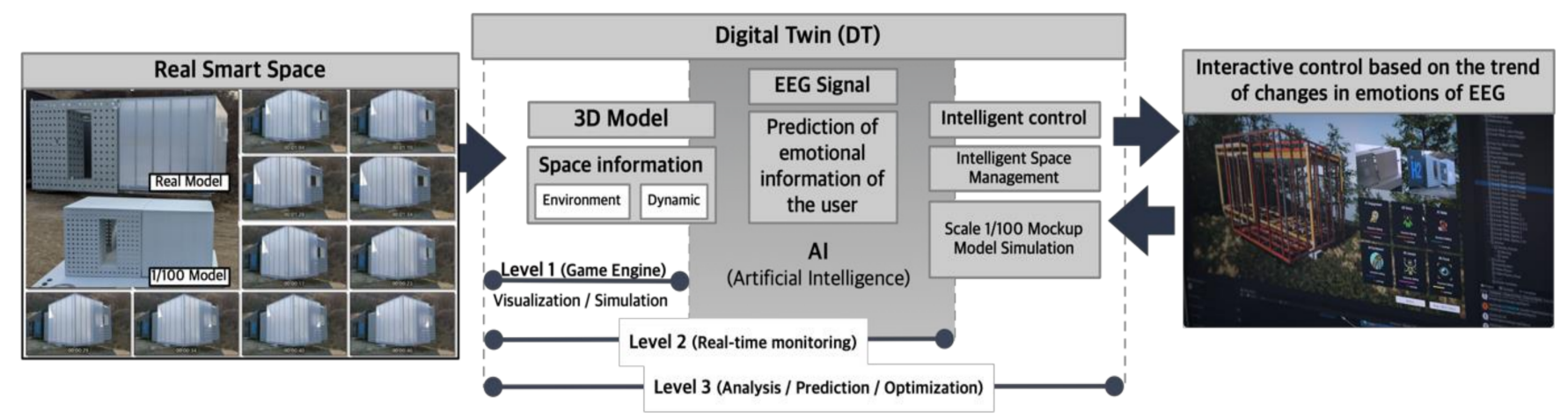

Figure 4. Digital twin (DT) and artificial intelligence (AI) integration mechanism.

By establishing an EEG information management and space control platform, it is possible to identify emotion information from EEG under various situations and operate an interactive space control process. In the EEG information management and space control platform, the data-collection process is performed to understand the context of 
the smart space control system. The collected data are analysed, processed, and saved in the information management and space control platform as the output of the space control system control command or as theoretical rules. The user's EEG information, which is collected according to the management and control of the space control system, is utilised as training data for a deep learning algorithm to identify emotional information; subsequently, judgement information for the diagnosis and prediction of the space control system responding to the emotional information is created. The main function is to collect, process, and analyse information. Quantitative values for the diagnosis of the emotional state of the user and the dimension control of the space are derived to control and manage the operation of the smart space control system. The key function of the information management and control platform is the fusion and integration of EEG information from human and machine-generated information. Fusion refers to managing data based on the same time series for the user's emotional state, which is affected by the space and the mechanical current status information of the space control system. Integration refers to integrating the data fused based on the time series, and the sub-item processes related to the EEG equipment and the motor for interactive control are integrated into the smart space so that the operation for inducing a positive emotional state of the user is ensured. The DT plays the role of a multiple safety device for rational operation; in this study, a virtual 3D model and a 1/100 scale model served as simulation models for rational operation in the same environment for the operation of a real building and performed the function of transmitting senses. In the information management and control platform, for the action of the active space change system, the information judged by AI through a mutual information transmission process between the virtual and real objects of DT is transmitted. The diagnosis and prediction information generated by the AI-based deep learning algorithm is transmitted to the interactive motor in real space through the DT system, forming a data feedback loop and realising the precise execution of virtual control and real control processes. The data feedback loop formed by the EEG information management and smart space control platform is depicted in Figure 5.

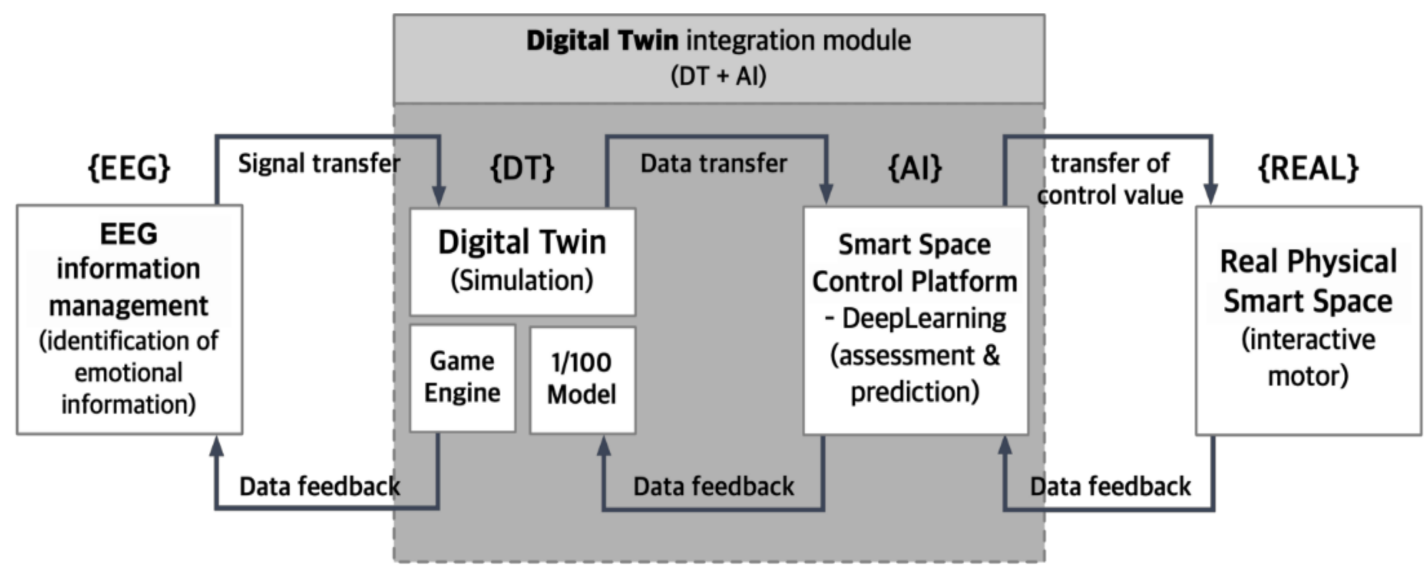

Figure 5. Schematic of feedback loop formed by EEG information management and smart space control platform.

The deep learning algorithm performs the key functions of supporting intelligent diagnosis before and after the operation of the space control system, in consideration of EEG data and prediction of the user's emotional state after the space change, as well as data analysis and data processing on the biometric information management and control platform. In this study, based on the deep learning algorithm, training sets were constructed, and a deep learning model was trained with a smart space control process structure by utilising the association that the EEG-based emotional information of the user is affected by the environmental elements of the space. Using EEG-based biometric data consisting of training sets, an analysis algorithm architecture was constructed for the change of emotional information of EEG according to space before and after space change. The collected EEG 
data of the user, environmental data of the space, and the simulation data were used as resources for the deep learning model training, eventually for the prediction performance for the active space change. By predicting space change with EEG information of smart space users, a game-engine-based integrated data model was constructed, which integrated data collected in the past, simulation data, and prediction data. Through the data model, the suggestion on the optimal space according to the user's emotions, prediction of the user's emotional state before and after the change of space, and review of the safety aspects in the process of mechanical space transformation were simulated and addressed, and the results were linked with DT to support intelligent diagnosis and prediction.

The virtual digital space in the game engine environment and the 1/100 mock-up model served as DT for data recognition and fusion. In DT, EEG data can be collected according to the user's real-time situation, and visualisation can be performed by considering the conditions of the real physical environment. In addition, DT performs essential control through repetitive training in the part where control of space occurs, and the anxiety factors that occur in the model can be identified and processed. The smart space control system driven through the analysis of the fusion mechanism of DT and AI technology is illustrated in Figure 6.

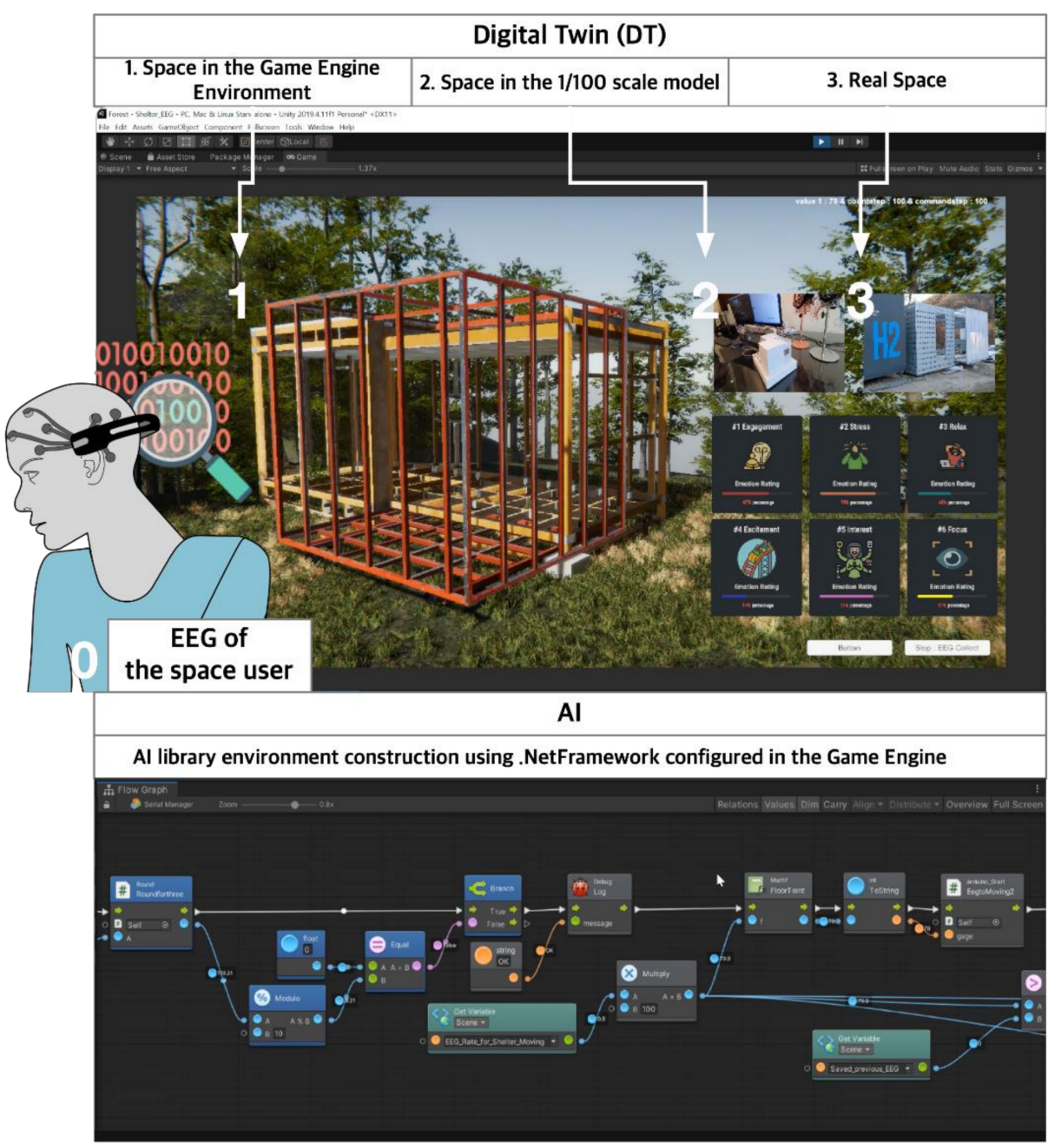

Figure 6. Fusion mechanism of DT and AI technologies.

\subsection{Deep Learning Agent for Intelligent Motor Control in Smart Space}

Through the process of dimensional expansion and reduction according to the EEG of smart space, DT provides the link between the physical world of the real space and the emotional information of the user utilising the virtual reality space. DT and AI are integrated 
in operation for collection, processing, and analysis of data generated in smart space, and deep learning algorithms are utilised for the simulation and diagnosis of human-machine interaction. It is important to construct a deep learning agent in connection with DT for automated processing, which leads to a sustainable improvement in the performance of a model by linking the deep learning algorithm with DT. Therefore, the introduction of the deep learning algorithm can improve the accuracy and efficiency of the physical system in the operation of smart space; moreover, the effectiveness of the utilisation of the user's biometric information can be examined through comparison of the EEG data before and after the space change. For the integrated operation of DT and AI in the process of changing the spatial dimensions, the physical space for interaction with smart space, sensor information, and construction of deep learning agent for biometric information are necessary prerequisites. The user-centred intelligent biometric information monitoring technology was developed based on the assumption that the appropriate change in the space induces psychological stability, a creative original approach to construct an AI model, and the pathology standard of Chombard de Lauwe. Using intelligent technology considering the EEG and space, DT technology with sufficient consideration of temporal and spatial dimensions is integrated into the spatial dimension, and intelligent and comprehensive analysis is performed on the component units according to the psychological stability and dimension of the space, and the value of EEG by channel to construct the configuration to achieve spatial dimensions with positive impact. Through environmental variables of the space, based on EEG values that represent the psychological information of the user according to the dimension of the space and the spatial dimensions for the space change are calculated. Provided that, for safety in the process of space transformation, the maximum and minimum space transformation dimensions are set in accordance with the mechanical tolerance values, the scale dimensions for movement are calculated. By indexing the stationary state, the initial state of the smart space, and the state with the maximum transformation based on percentiles, deep learning algorithms process the data cleansing to produce data for easy operation. This is a compensation concept for inducing positive emotions after spatial transformation for the effective operation of the deep learning model in a smart space. If the difference in the user's EEG-based psychological index is positive when comparing data before and after the spatial change, it is compensated; however, when the index is negative, loss is assigned such that it can be used as reference data for inducing active performance improvement of the AI model according to the interactive space change between the EEG and space. The cooperative operation mechanism between DT and AI based on the deep learning agent is established to support intelligent smart space construction process modelling to achieve psychological stability of users in the domain of time information and space transformation. In this study, DeepFM was selected as the deep learning algorithm.

The main features of DeepFM used in this study were to identify the data feature points and relationships of the input variables, to estimate the CTR value, and to recommend items with high CTR. In the system, the one with a high CTR value is the value of the change in the smart space. In the application of the DeepFM algorithm in this study, EEG information and the status information of the space were listed in the time series, and the deep learning model was trained; based on the real-time context information, the optimal control value was recommended. The point where the psychological stability difference is positive was recommended as CTR, and the smart space control value was recommended through the algorithm based on the current psychological index value. Thus, for the integrated control of the virtual and real spaces, the multiple environmental elements that constitute the space, the simulation of a parallel structure for the emotional state according to the user's EEG, and the smart space construction system that considers the EEG and smart spatial dimensions were used. For the implementation of the interactive space between the user's biometric information and machine, the deep learning agent was constructed using the DeepFM algorithm, as shown in Figure 7. 

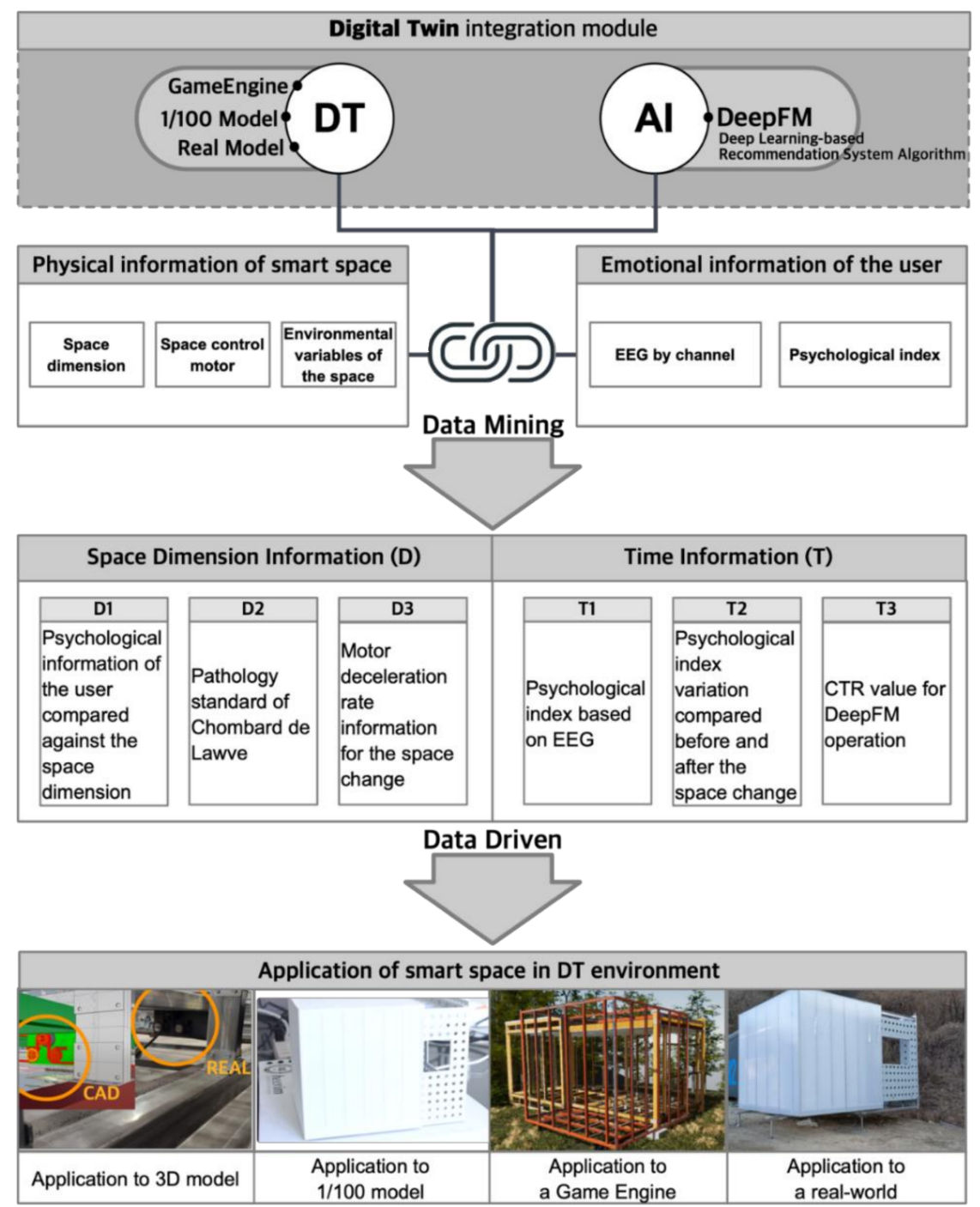

Figure 7. Composition of deep learning agent for smart space control.

\subsection{Fusion of DT and AI for Optimisation of Intelligent Motor Control in Smart Space}

With DeepFM-based AI model for effective operation of the motor system of smart space based on EEG, the work of moving the space with a linear actuator type motor and the safety review using the sensor information that transmits the status of the building during the move were conducted by dividing them into real, semi-real, and virtual cases, and the corresponding data were received and converged through DeepFM. The datacollection process for sensor data for the motor operation process in the real environment was performed explicitly; in the semi-real case, which uses the 1/100 scale model, implicit processes were constructed for motor operation using the sensor, motor information, and physics engine of the game engine, and sensor data processing for stability. In particular, in the virtual case, which uses the game engine, based on the information collected in the real and semi-real cases, the collision event that utilises the functions of collider and trigger that are used for reproduction of realistic games was used as the sensor, which is the key function for DT implementation, was used in connection with DeepFM (Figure 8). 


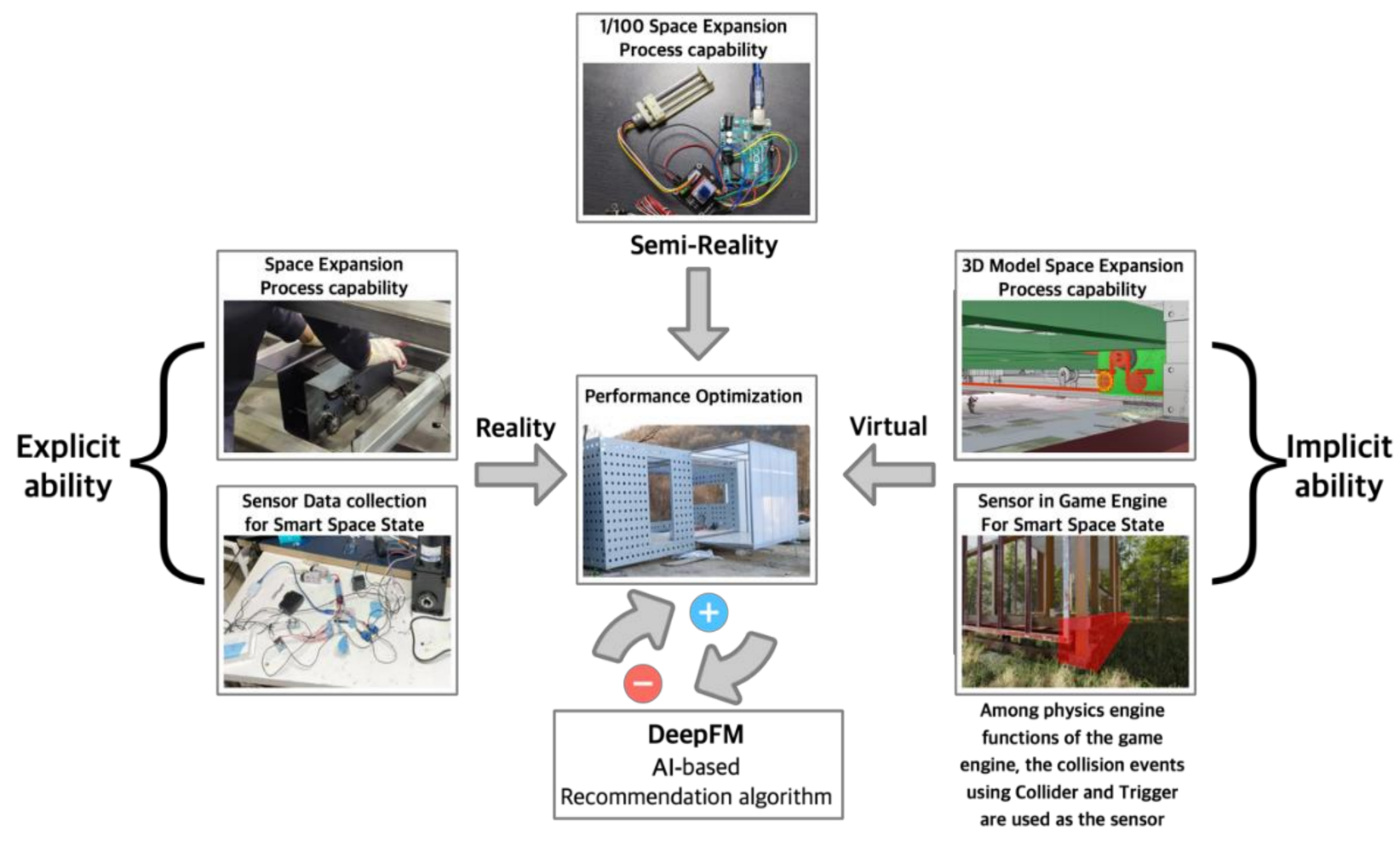

Figure 8. Performance optimisation of motor system for smart space based on DT.

Explicitability is a smart space that is in operation for real, and implicitability involves performing simulation to induce the correct operation of the smart space based on EEG. Implicitability is performed based on the game engine, and the validation is performed through a model. Here, DeepFM performs the role of converging the status information of the three situations according to DT and conducts the operation and judgement. In this study, DeepFM recommended the distance value that the space moves according to the scale of the space expansion. In particular, the EEG data per channel, emotional information using the AI model that converts the EEG of Emotiv to emotional metric, and smart space environmental status were utilised as information resources.

In this study, the data collected for the operation of the smart space was limited to the data calculated from the EEG device worn by the user in the space. The EEG data and gyro sensor information, which can detect a change in the user's position, were used. In this manner, as DeepFM was utilised to identify the potential causal relationship between the space and the user's EEG data, it was necessary to estimate the CTR value to determine whether the difference in the emotional index corresponding to the stress has increased or decreased after the space is changed. If the stress index value rises after the change, the changed space is recommended. After the first operation through the 1/100 model, it is reflected in the real building. To improve the above process, based on the estimated CRT value obtained using the stress change difference before and after the change in space, DeepFM recommends a range of space expansion.

Figure 9 shows the data structure for operating the DeepFM algorithm with the integrated operation of DT and AI. In the case of DeepFM, based on time information, information on the emotion of the user in the smart space is organised by assigning the attributes as low-order and high-order features. With columns according to the number of horizontal arrays, the user information, EEG, game engine physics simulation information, gyro sensor, time, and EEG-based emotional metric are collected in terms of number of seconds. The collected data receive the motor control values for optimal space expansion to Target y through the interaction mechanism and training of the DT and the DeepFM algorithm. 


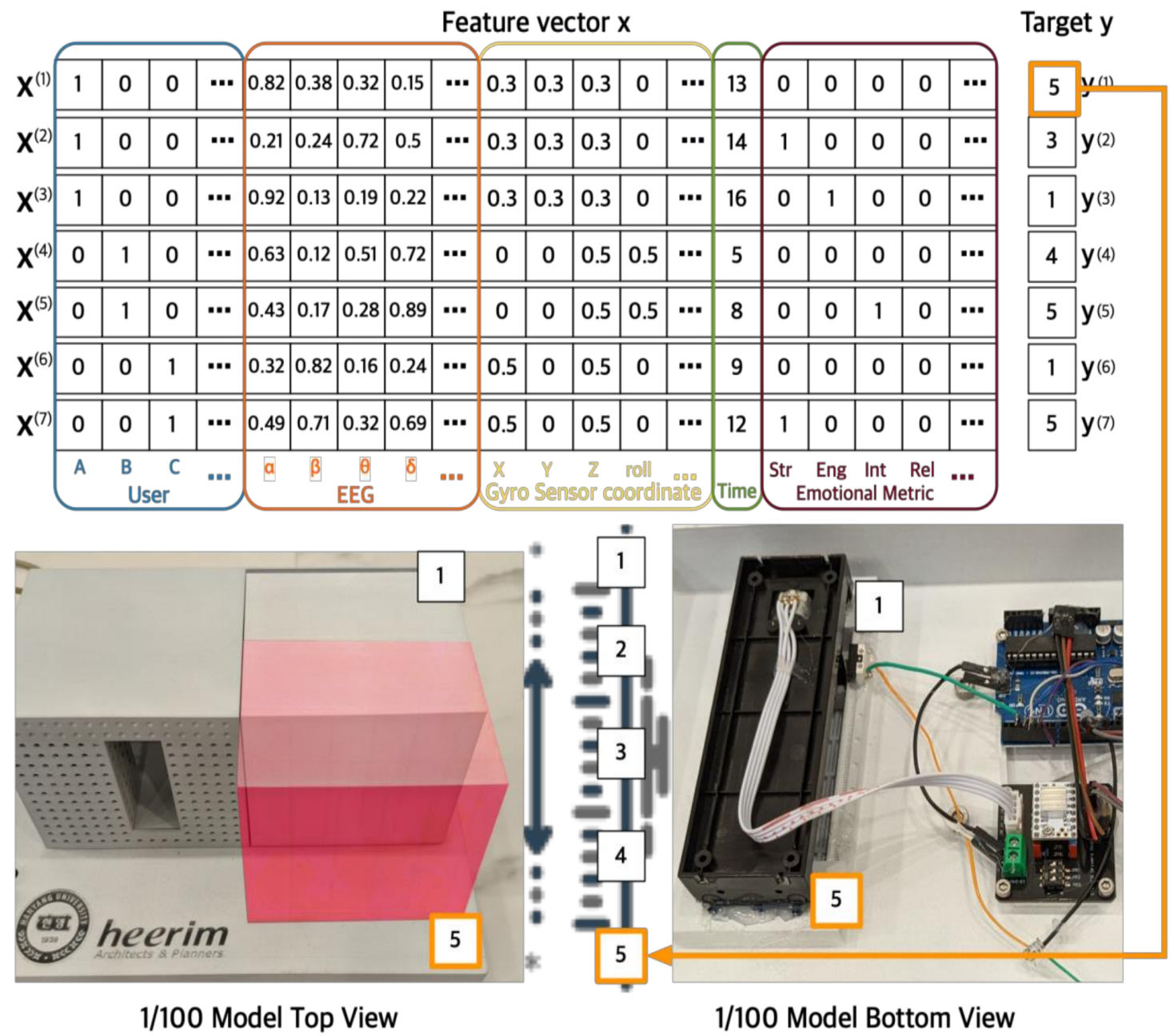

Figure 9. Smart space operation with application of DeepFM algorithm (input).

CTR prediction was performed through DeepFM, and for the recommended Target $y$ value, first, the smart space of the game engine was operated, and the EEG noise was detected through physics operation of the game engine, and the movement value was remapped within the range between 1 and 5 specified in Target $y$. The Remap function was applied to each part of the DT by applying the method used in programming languages such as JavaScript or C\#, in which the number pattern of one range is remapped to the number pattern of a different range. After applying the data verified through the game engine to the small motor of the $1 / 100$ scale model, if there was no abnormality in operation, it was applied to the real building. The working process is illustrated in Figure 9.

\section{Smart Space Size Control Run by EEG-based DT and AI Integration for a Single User}

The interactive motor control of smart space is a complex and systematic project involving a combination of environmental factors, technology, and multi-dimensional characteristics, and the user's EEG data changes in real-time owing to various and uncertain factors. The linear actuator type interactive motor control system was operated by an analogue DC motor, and thus it is dynamic and has non-linear characteristics. Therefore, the system cannot be accurately operated without an auxiliary sensor, which makes the application of DT effective. Although this type of a motor shows reasonable power to manipulate heavy objects, it is highly likely to become unstable when one tries to elaborately control it, using EEG-based emotion information. In this study, the author's EEG data was collected since 2012; thus, the EEG data model for deriving emotion information is as complete as possible. Subsequently, considering the safety issue of operating a building 
weighing close to 1 tonne, the work of optimising the operation of the smart space for a single person alone was conducted.

The following are the main components of the proposed system:

1. With the same situational information for all factors of the intelligent motor control process of the smart space as the real building, a 3D model was constructed in the game engine along with a 1/100 scale model. Based on the user's emotional information in space, three parts corresponding to $\mathrm{DT}^{\prime}$ s real, semi-real, and virtual cases were composed, and data processing and analysis was performed for EEG data of environmental components across the entire smart space and the user data for the space environment. In addition, in the process of space expansion and contraction, through real-time collection of user information, the capturing and mining of the EEG data before space expansion was performed. In addition, based on the EEG history information of one person that was learned in advance, in the process of smart space control to achieve an emotionally stable state in EEG, the cumulative data were collected and captured in the local storage of the game engine.

2. In terms of the feedback control between the EEG and interactive motor of the smart space control process, the problem of decreasing accuracy due to non-linear motion of the linear actuator motor in actual operation was resolved by using a physical sensor and collision events utilising Trigger and Collider, among the physics engine of the game engine, as the sensor, and applying it to DT. Thus, the fusion mechanism between the virtual space and the real space system was established. The interaction between the real, semi-real, and virtual cases and the feedback control process in connection with EEG also played the role of a safety device for the final real operation.

3. In terms of the optimisation of the smart space control process and the integration mechanism of the control system through EEG, a fusion mechanism between DT and AI was constructed using DeepFM. Based on the difference in the psychological index before and after the space change in the system that controls the expansion of the smart space, a model that recommends the CTR prediction value for optimal expansion was constructed. In this way, the values of the optimal space movement variables were derived under uncertain and complex conditions based on the context.

4. In terms of the decision-making level of optimisation management and control of the entire process of smart space control, the DeepFM algorithm was used to promote the overall data mining and knowledge accumulation of the recommendation system for a series of patterns, such as in the case of a user's high stress level, the space was expanded to decrease the stress level. However, when the user had a low level of engagement, the space was contracted to enhance the engagement. The overall operation status and trend were diagnosed and predicted, and the abnormal operation status according to the noise of the EEG was detected through the game engine and the $1 / 100$ scale model. Thus, the load on the motor in the real space was reduced, and operations based on the stability of the emotional metric of the user was induced.

Using the proposed system, an interaction experiment was conducted using the emotional information of the EEG sensor and smart space with a single subject.

The Figure 10 is the space used for the experiment, and it stretches up to $7000 \mathrm{~m}$ according to the influence of EEG.

Based on the analysis of the space change according to the recommendation of the real space, this method was applied to the real-space structure as shown in Figure 11.

As shown in Table 1, first, the CTR(c) was set to the pre-set value. As the rate of increase in engagement increases, the range of CTR(c) converges closer to 0. Similarly, as the rate of stress decrease increases, the range of CTR(c) converges closer to 1 . 


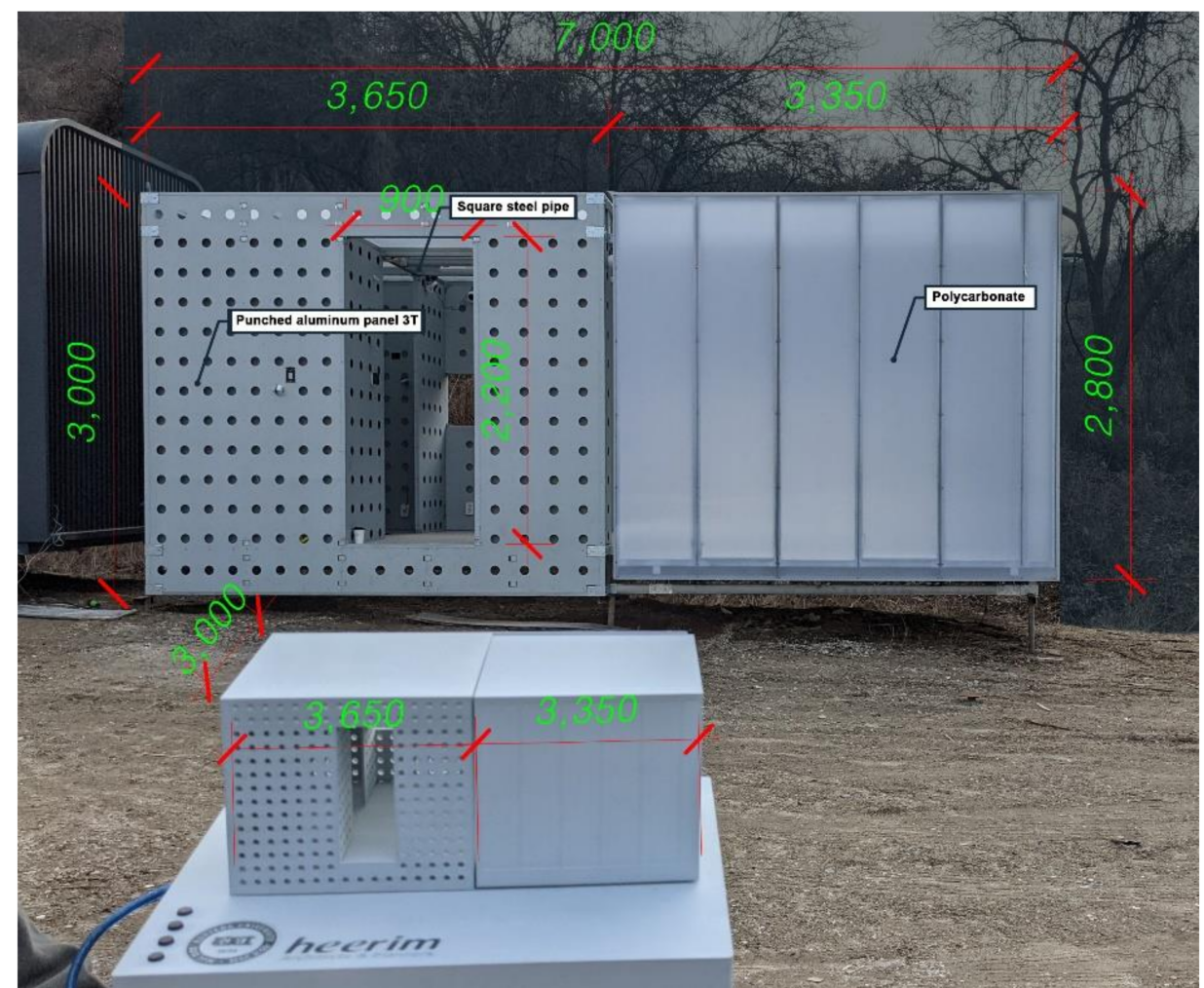

Figure 10. Real-Smart space materials and dimensions.

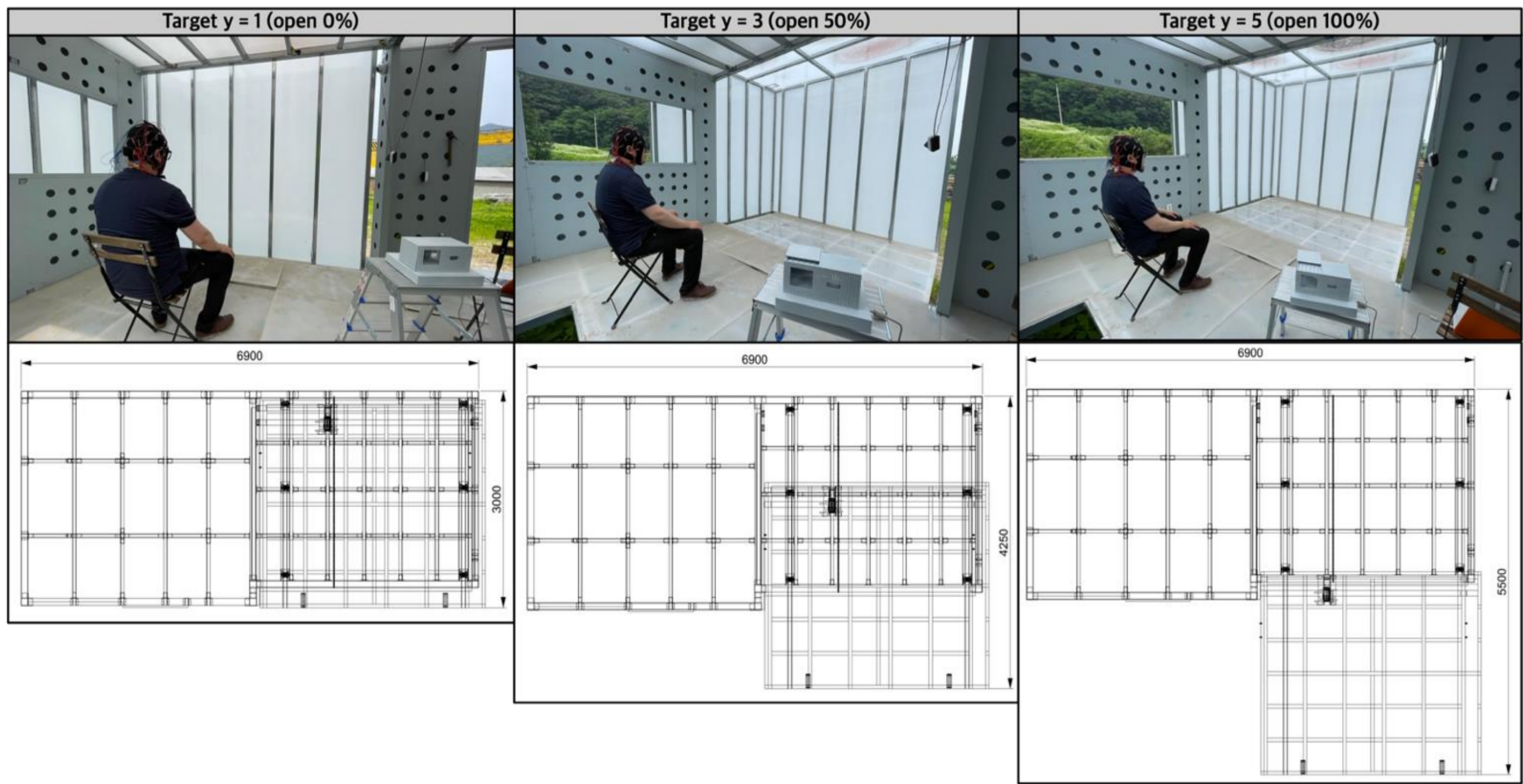

Figure 11. The process of applying the method to the real space structure.

CTR prediction was performed using DeepFM, and based on the range of the predicted value, the point at which the smart space moves was designated to be between Nos. 1 and 5 for recommendation. A total of 10 experiments were conducted for $10 \mathrm{~min}$ each, and after applying the user's EEG to DeepFM according to the space situation, the three cases of DT- virtual, semi-real, and real, were applied sequentially. Fifty-nine space transformations were performed in a total of $100 \mathrm{~min}$. After the space change, the recom- 
mendation accuracy of DeepFM for space transformations that induce high engagement or low stress after space transformation was found to be $50 \%$. The accuracy was highest when the spatial dimensions of No. 1 were recommended. As for the accuracy of lowering stress compared with the number of recommendations, No. 4 showed the highest accuracy (Table 1).

Table 1. Accuracy in number and rate according to increase in engagement and decrease in stress compared to the number of recommendations by AI.

\begin{tabular}{|c|c|c|c|c|c|c|}
\hline $\begin{array}{c}\text { Smart Space } \\
\text { Recommendation } \\
\text { Item by CTR } \\
\text { Prediction }\end{array}$ & CTR(c) & Spatial Dimensions & $\begin{array}{l}\text { Number of Cases } \\
\text { Inducing an } \\
\text { Increase in } \\
\text { Engagement } \\
\text { (Number of } \\
\text { Recommendation) }\end{array}$ & $\begin{array}{l}\text { Accuracy of the } \\
\text { Number of Cases } \\
\text { inducing an } \\
\text { Increase in } \\
\text { Engagement } \\
\text { Compared with the } \\
\text { Recommendation }\end{array}$ & $\begin{array}{l}\text { Number of Cases } \\
\text { Inducing a } \\
\text { Decrease in Stress } \\
\text { (Number of } \\
\text { Recommendation) }\end{array}$ & $\begin{array}{l}\text { Accuracy of the } \\
\text { Number of Cases } \\
\text { Inducing a } \\
\text { Decrease in Stress } \\
\text { Compared with the } \\
\text { Recommendation }\end{array}$ \\
\hline 1 & $0 \leq \mathrm{c} \leq 0.2$ & $9 \mathrm{~m}^{2}$ & $3(6)$ & $50 \%$ & $1(6)$ & $16 \%$ \\
\hline 2 & $0.3 \leq \mathrm{c} \leq 0.4$ & $10.8 \mathrm{~m}^{2}$ & $7(15)$ & $47 \%$ & $2(15)$ & $13 \%$ \\
\hline 3 & $0.5 \leq \mathrm{c} \leq 0.6$ & $12.6 \mathrm{~m}^{2}$ & $3(11)$ & $27 \%$ & $4(11)$ & $36 \%$ \\
\hline 4 & $0.7 \leq \mathrm{c} \leq 0.8$ & $14.4 \mathrm{~m}^{2}$ & $4(9)$ & $44 \%$ & $8(9)$ & $88 \%$ \\
\hline 5 & $0.9 \leq \mathrm{c} \leq 1$ & $16.2 \mathrm{~m}^{2}$ & $3(18)$ & $16 \%$ & $13(18)$ & $72 \%$ \\
\hline
\end{tabular}

The average change rate when the concentration was higher after the space change than before was $15 \%$ in the case of No. 1, and this was the highest rate in the experiment. After the space change, the average change rate when the stress was lower than before was the highest in the case of No. 5 at $-18 \%$ (Table 2).

Table 2. Average rate of change upon success in increasing engagement and lowering stress after space change by AI recommendation.

\begin{tabular}{ccccc}
\hline $\begin{array}{c}\text { Smart Space } \\
\text { Recommendation } \\
\text { Items by }\end{array}$ & CTR(c) & Spatial Dimensions & $\begin{array}{c}\text { Average Change Rate } \\
\text { of Engagement after } \\
\text { Space Change } \\
\text { (In Case of Success in } \\
\text { Inducing an Increase } \\
\text { in Engagement) }\end{array}$ & $\begin{array}{c}\text { Average Change Rate } \\
\text { of Stress after } \\
\text { Space Change } \\
\text { (In Case of Success in } \\
\text { Inducing a Decrease } \\
\text { in Stress) }\end{array}$ \\
\hline 1 & $0 \leq \mathrm{c} \leq 0.2$ & $9 \mathrm{~m} 2$ & $15 \%$ & $7 \%$ \\
\hline 2 & $0.3 \leq \mathrm{c} \leq 0.4$ & $10.8 \mathrm{~m} 2$ & $6 \%$ & $5 \%$ \\
3 & $0.5 \leq \mathrm{c} \leq 0.6$ & $12.6 \mathrm{~m} 2$ & $5 \%$ & $-2 \%$ \\
5 & $0.7 \leq \mathrm{c} \leq 0.8$ & $14.4 \mathrm{~m} 2$ & $-2 \%$ & $-10 \%$ \\
\hline & $0.9 \leq \mathrm{c} \leq 1$ & $16.2 \mathrm{~m} 2$ & $-2 \%$ & $-18 \%$ \\
\hline
\end{tabular}

Generalisation of the results of this experiment, which was performed with one subject, may not be reasonable. The method of fusion of DT and AI is a novel and significant contribution of this study in which, based on the EEG of the user, the AI algorithm to lower the stress level or increase the engagement of the user by changing the space is linked with DT.

\section{Conclusions}

In this study, DT and AI were utilised for efficient management and control of a smart space expansion system based on the user's EEG. Furthermore, in the case of architecturebased DT, the same space structure and the motor for expansion and contraction of the smart space were implemented as in a real building, 1/100 scale model, and the game engine, thereby creating a space that makes use of the user's emotional state to induce a positive emotional state. In particular, based on the pathology standard of Chombard de Lauwe, while wearing an EEG device and using the basic rule of expansion of space for lowering stress when the stress-related EEG value increases, and vice versa, the interactive process for expansion and contraction of the smart space was designed. This study also analysed the current status of research and information technology applications in the 
field of architecture using EEG and investigated the fusion mechanism of DT and AI. Furthermore, to implement an intelligent decision-making process based on the emotional information for each situation, the fusion of DT and AI was introduced, and a DeepFMbased recommendation system algorithm was analysed and applied. It can be summarised as follows:

1. The fusion mechanism of DT and AI was considered with the aim of applying DT at the stage of 'reflecting the virtual model in the game engine and 1/100 scale model to the real space'. The combination of the two technologies provides new ideas for the realisation of 'recognising the status of smart space, real-time data processing, intelligent diagnosis and decision-making' to reflect the user's EEG and induce positive emotions.

2. To apply the fusion mechanism of DT and AI to the smart space, first, based on the spatial dimension information about the smart space and time information, the user's psychological information compared to the dimension of the space, the emotional metric difference before and after the change of space, the pathology standard of Chombard de Lauwe, the motor deceleration ratio information for space transformation, and the CTR prediction through DeepFM operation, the foundation for intelligent diagnosis, AI-based spatial dimension prediction, and accurate execution were established. Based on the DT and AI models, in-depth integration capabilities to improve the overall optimised decision-making of smart space systems were explored.

3. The interactive motor control that reflects the emotional state of a smart space requires systematic management because the space is used by human users. To minimise the malfunction of the motor owing to data bouncing according to simple signal processing between the operation of EEG in a complex environment of interference with the AI control centre as the core in space expansion, the framework in which the performance is continuously improved through data feedback between each role of DT was implemented.

4. With the aim of inducing positive emotions through intelligent space control for a single user, a DeepFM algorithm was utilised to achieve virtual and actual interaction using a linear actuator type interactive motor driven by DT and AI fusion as well as EEG technology to detect the emotion of the user. In the integrated model of DT and $\mathrm{AI}$, real space, scale model space, and virtual spaces were categorised as real, semireal, and virtual, respectively, and each part implemented interactive control between the machine and the user's biometric data. For a stable and accurate operation of the real part, the AI algorithm operation and safety filtering was performed in the virtual and semi-real parts, and a scaled version of the motor and space expansion structure was constructed to conduct safety validation.

In general, by applying DT and AI, positive emotions can be induced through the EEG of users by utilising the routine of space expansion and contraction in a smart space, and the method has a wide range of applicability. However, as in the case of the implementation of DT, research on EEG-based brain waves and architectural space is still in its early stages. Only DT-centred linking in connection with mechanical equipment has been conducted through the phenomenological investigation of EEG. By improving the method of information collection and communication technology through sensors to analyse the space situation and real-time analysis of the user's emotions for the space, in-depth integration of DT with AI and the latest edge technology can be achieved. In addition, ongoing research on intelligent construction and management for all elements of the entire process is required. Future studies will focus on improving and supplementing the above issues and further exploring the relationship between architectural space and EEG. 
Funding: This work was supported by a National Research Foundation of Korea (NRF) grant funded by the Korean government. (2019R1C1C1008187).

Institutional Review Board Statement: The study was conducted according to the guidelines of the Declaration of Helsinki, and approved by the Institutional Review Board of Hanyang University(HYUIRB202110-007, 2021.10.22)

Informed Consent Statement: Informed consent was obtained from all subjects involved in the study

Conflicts of Interest: The authors declare no conflict of interest.

\section{References}

1. Xu, L.D.; Xu, E.L.; Li, L. Industry 4.0: State of the art and future trends. Int. J. Prod. Res. 2018, 56, 2941-2962. [CrossRef]

2. Kourtit, K.; Nijkamp, P. A smart cities in smart space: A regional science perspective. Sci. Reg. 2017, 1, $105-114$.

3. Filipponi, L.; Vitaletti, A.; Landi, G.; Memeo, V.; Laura, G.; Pucci, P. Smart city: An Event Driven Architecture for Monitoring Public Spaces with Heterogeneous Sensors. In Proceedings of the 4th International Conference on Advanced Information Technology and Sensor Application; ACM Library: New York, NY, USA, 2010; pp. 281-286.

4. Familiar, M.S.; Martínez, J.F.; López, L. Pervasive smart spaces and environments: A service-oriented middleware architecture for wireless ad hoc and sensor networks. Int. J. Distrib. Sens. Netw. 2012, 8. [CrossRef]

5. Morita, M.; Shinoda, Y. Information Filtering Based on User Behavior Analysis and Best Match Text Retrieval. Acad. Med. N. Y. SIGIR '94 1 August 1994. 1994, 272-281.

6. Broom, A. Using qualitative interviews in CAM research: A guide to study design, data collection and data analysis. Complement. Ther. Med. 2005, 13, 65-73. [CrossRef] [PubMed]

7. Szajerman, D.; Napieralski, P.; Lecointe, J.P. Joint analysis of simultaneous EEG and eye tracking data for video images. COMPEL Int. J. Comput. Math. Electr. Electron. Eng. 2018, 37, 1870-1884. [CrossRef]

8. Bleichner, M.G.; Lundbeck, M.; Selisky, M.; Minow, F.; Jäger, M.; Emkes, R.; Debener, S.; De Vos, M. Exploring miniaturized EEG electrodes for brain-computer interfaces. An EEG you do not see? Physiol. Rep. 2015, 3, e12362. [CrossRef] [PubMed]

9. Eberhard, J. Brain Landscape: The Coexistence Neuroscience and Architecture; Oxford University Press: New York, NY, USA, 2009.

10. Sternberg, R.J. Inside intelligence: Cognitive Science enables us to go beyond intelligence tests and understand how the human mind solves problems. Am. Sci. 1986, 74, 137-143. 